\title{
Study of Protoscolicidal Effects of Hypertonic Glucose on Protoscolices of Hydatid Cyst at Different Concentrations and Exposure Times
}

\author{
Mojtaba Shahnazi, ${ }^{1,2}$ Fatemeh Badakhsh, ${ }^{1}$ Abbas Azadmehr, ${ }^{3}$ Mehrzad Saraei, \\ Mahmood Alipour, ${ }^{4}$ Maryam Shahnazi, ${ }^{5}$ and Mehri Jamshidii \\ ${ }^{1}$ Department of Parasitology, Qazvin University of Medical Sciences, Qazvin, Iran \\ ${ }^{2}$ Cellular \& Molecular Research Institute, Qazvin University of Medical Sciences, Qazvin, Iran \\ ${ }^{3}$ Department of Immunology, Qazvin University of Medical Sciences, Qazvin, Iran \\ ${ }^{4}$ Department of Social Medicine, Qazvin University of Medical Sciences, Qazvin, Iran \\ ${ }^{5}$ Zanjan University of Medical Sciences, Zanjan, Iran \\ ${ }^{6}$ Department of Pediatrics, Ghods Hospital, Qazvin University of Medical Sciences, Qazvin, Iran
}

Correspondence should be addressed to Mehri Jamshidi; shahnazi58@yahoo.com

Received 21 April 2014; Revised 13 September 2014; Accepted 15 September 2014; Published 30 October 2014

Academic Editor: Atilla Arslan

Copyright (C) 2014 Mojtaba Shahnazi et al. This is an open access article distributed under the Creative Commons Attribution License, which permits unrestricted use, distribution, and reproduction in any medium, provided the original work is properly cited.

\begin{abstract}
Surgical operation is the treatment of choice for hydatid cyst. To date, many protoscolicidal substances have been used for inactivation of hydatid cyst contents but most of these compounds may produce several side effects. The aim of this study was to evaluate the protoscolicidal effects of hypertonic glucose on protoscolices of hydatid cyst at different concentrations and exposure times. Protoscolices were obtained aseptically from the livers of slaughtered sheep at Qazvin abattoir, Iran. Protoscolices were exposed to different concentrations of hypertonic glucose $(10 \%, 15 \%, 20 \%, 25 \%, 30 \%, 40 \%$, and $50 \%)$ at different times $(1,2,3,4,5$, $6,7,8,9,10,20,30,40,50$, and $60 \mathrm{~min})$. Viability of protoscolices was evaluated by $0.1 \%$ eosin and the movement of protoscolices flame cells. The highest protoscolicidal effect (100\%) of hypertonic glucose was obtained at concentrations $40 \%$ and $50 \%$ following 40 and 20 min exposure times, respectively. Some protoscolicidal agents show a variety of dangerous complications such as biliary tract fibrosis and liver necrosis; however, hypertonic glucose especially at a concentration of $40 \%$ may demonstrate less side effects compared with glucose $50 \%$. Further in vivo investigations are suggested.
\end{abstract}

\section{Introduction}

Hydatidosis is one of the most important zoonotic diseases with worldwide distribution. Although hydatid disease is mostly found in liver and lung, it can arise anywhere in the body [1]. Currently, there are three options for the treatment of liver hydatid disease, where, among them, surgical operation is considered as the most efficient treatment. [2, 3]. Spillage of the cyst contents is very common, despite taking technical precautions. This is the major cause of recurrence, which is seen in approximately $10 \%$ of postoperative cases. During the operation and discharging of the cyst, the injection of protoscolicidal agents into hydatid cyst is performed to prevent the spread of infection and secondary cyst formations $[2,4,5]$. To date, many protoscolicidal substances (formalin, hydrogen peroxide, cetrimide, pure alcohol, hypertonic saline, and silver nitrate) have been used for inactivation of the hydatid cyst contents; nevertheless most of such compounds may produce a variety of side effects $[3,6,7]$. Hypertonic glucose is a cheap and available material, and due to the possibility of intravenous injection, it is with low complication and a trusted substance [8]. In addition, hypertonic glucose is reported to be a successful protoscolicidal agent [8-12]. So far, the protoscolicidal efficacy of 
hypertonic glucose at different concentrations and exposure times has not been tested by in vitro studies. Therefore, this study was undertaken to evaluate the protoscolicidal effects of hypertonic glucose on protoscolices of hydatid cyst at different concentrations and exposure times.

\section{Materials and Methods}

2.1. Collection of Protoscolices. The fluid of hydatid cysts was gathered aseptically from the livers of slaughtered sheep at Qazvin abattoir, in the central of Iran. Fertility of the cysts was assessed and protoscolices of hydatid cysts were washed three times in normal saline. Viability of protoscolices was confirmed prior to the experiments. The viability of protoscolices was determined by their motility characteristics and with $0.1 \%$ eosin staining under light microscopy. When the percentage of viable protoscolices was greater than $90 \%$, they were considered to be appropriate for our study.

2.2. Scolicidal Assay. In this study, the protoscolices were exposed to different concentrations of hypertonic glucose $(10 \%, 15 \%, 20 \%, 25 \%, 30 \%, 40 \%$, and $50 \%)$ at room temperature for different times $(1,2,3,4,5,6,7,8,9,10,20,30,40$, 50 , and $60 \mathrm{~min})$. Briefly, in each experiment, $2.5 \mathrm{~mL}$ of each concentration of hypertonic glucose was placed in a test tube, to which a drop of protoscolex-rich sediment (containing at least 1000 protoscolices) was added using a Pasteur pipette. The content of each tube was gently mixed and incubated. At the end of each incubation time $(1,2,3,4,5,6,7,8,9,10,20,30$, 40,50 , and $60 \mathrm{~min}$ ), $10 \mathrm{~mL}$ of normal saline was added to each tube and centrifuged for $1 \mathrm{~min}$ at $300 \mathrm{rpm}$ and the supernatant was discarded. The same procedure was repeated 6 times. One milliliter of $0.1 \%$ eosin stain was then added to the remaining settled protoscolices, mixed gently, incubated for $15 \mathrm{~min}$, and followed by careful removal of the upper portion. Later, the remaining protoscolices pellet was smeared on a manually scaled glass slide, covered with a cover glass, and examined under a light microscope. The percentages of dead protoscolices were determined by counting an average of 1000 protoscolices. Hypertonic saline (20\%) and normal saline were used as positive and negative controls, respectively. All experiments were performed six times $[5,13]$.

2.3. Statistical Analysis. Data are represented as mean \pm standard deviation. Statistical analyses were performed by one-way analysis of variance (ANOVA) and $t$-test to express the difference among the groups. All analyses were performed using SPSS software version 16. Data were considered statistically significant at $P<0.05$.

\section{Results}

The scolicidal effect of hypertonic glucose at different concentrations $(10 \%, 15 \%, 20 \%, 25 \%, 30 \%, 40 \%$, and $50 \%)$ and exposure times $(1,2,3,4,5,6,7,8,9,10,20,30,40,50$, and $60 \mathrm{~min})$ is shown in Table 1 . Hypertonic saline (20\%) and normal saline were used as positive and negative controls, respectively.

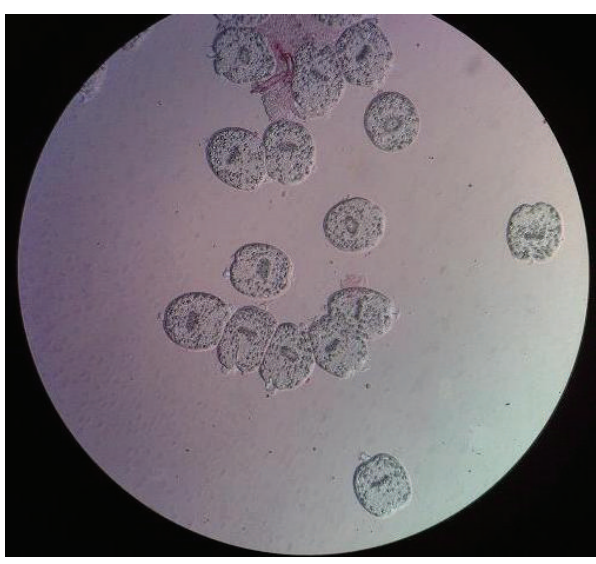

FIGURE 1: Live protoscolices after staining with $0.1 \%$ eosin.

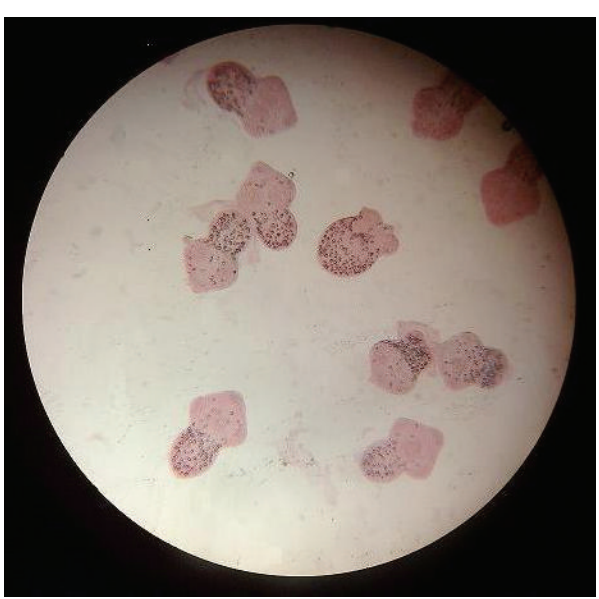

Figure 2: Dead protoscolices after 40 min exposure time with $40 \%$ glucose.

Glucose at $10 \%, 15 \%, 20 \%, 25 \%$, and $30 \%$ concentrations had a little effect on protoscolices after 1, 2, 3, 4, 5, 6, 7, 8 , 9, and $10 \mathrm{~min}$ exposure times. Concentrations of $10 \%$ and $15 \%$, even after 60 min exposure time, failed to show a considerable effect on protoscolices, but glucose at $20 \%$ and $25 \%$ concentrations after $40 \mathrm{~min}$ exposure time killed more than $50 \%$ of protoscolices. In addition, the mortality rates of $30 \%$ glucose following 30, 40, 50, and $60 \mathrm{~min}$ exposure times were $52.1 \%, 61.7 \%, 64.1 \%$, and $86 \%$, respectively. All protoscolices were killed after $40 \mathrm{~min}$ exposure time with $40 \%$ glucose as shown in Figure 1 (live protoscolices) and Figure 2 (dead protoscolices). The scolicidal effect of such glucose concentration after 20 and $30 \mathrm{~min}$ was $98.8 \%$ and $99.6 \%$, respectively. Glucose at $50 \%$ concentration, after 15 min exposure time, killed almost $50 \%$ of protoscolices, but after $6,7,8,9,10$, and 20 min exposure times, the scolicidal effect of this glucose solution was $63.9 \%, 67.2 \%, 83.4 \%, 95.3 \%$, $97.4 \%$, and $100 \%$, respectively. The results of this study showed that the scolicidal effect of all seven concentrations of glucose was significant comparing with the control groups at all exposure times $(P<0.001)$. Moreover, the protoscolicidal 


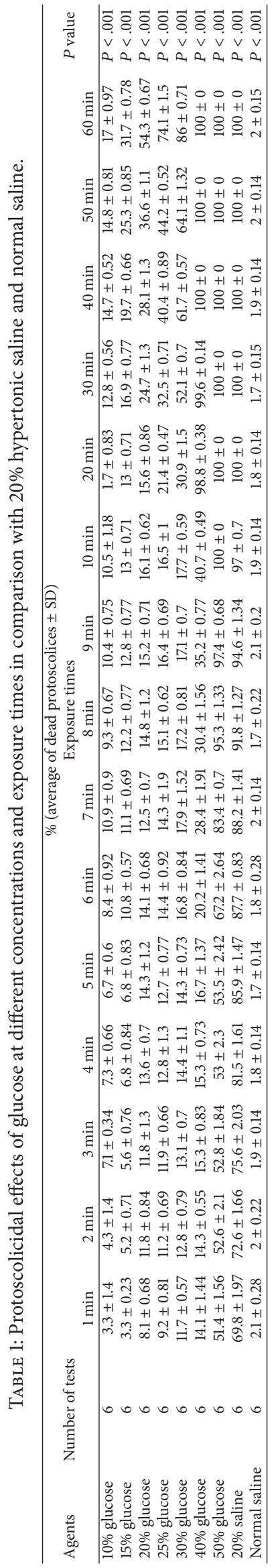


effects of different concentrations of glucose except for the $40 \%$ and $50 \%$ concentrations in various exposure times, compared with the effect of $20 \%$ hypertonic saline, were found to be significant $(P<0.001)$. Therefore glucose at concentrations of $40 \%$ and $50 \%$, like hypertonic saline $20 \%$, killed all (100\%) protoscolices after 40 and 20 min exposure times, respectively.

\section{Discussion}

In this study, the protoscolicidal effect of glucose at various concentrations was assessed at different exposure times and the results were statistically found to be significant compared with the control groups. The fatality rate of glucose at different concentrations on protoscolices was further examined by increasing the exposure times which demonstrated the effect of time on eliminating the viability of protoscolices. Moreover, our results showed that, at a fixed exposure time, increasing the glucose concentrations led to reduced viability of protoscolices, a finding in agreement with the results of other researchers $[5,14-16]$. Of course, glucose in some gradually increasing concentrations including $10 \%, 15 \%, 20 \%$, $25 \%$, and $30 \%$ and exposure times such as $1,2,3,4,5,6,7,8$, 9 , and $10 \mathrm{~min}$ produced a little effect on protoscolices with no significant difference. Meanwhile, other studies also have shown that glucose at $10 \%, 15 \%$, and $25 \%$ concentrations is without any obvious protoscolicidal effect after 1, 2, and $5 \mathrm{~min}$ exposure times [12]. Based on our results, glucose concentrations of $10 \%$ and $15 \%$, even after 60 minutes of exposure time, had no significant scolicidal effect on the protoscolices, implying the absence of glucose effect on protoscolices at low concentrations. Glucose at $20 \%$ and $25 \%$ concentrations after 60 minutes killed more than $50 \%$ of protoscolices, indicating that higher glucose concentrations following longer exposure times were more effective compared to lower glucose concentrations. The viability of about $50 \%$ and $85 \%$ of protoscolices disappeared after 30 and 60 min exposure times with $30 \%$ glucose, respectively. In addition, our results showed that glucose at $30 \%$ concentration could significantly eliminate the viability of protoscolices after $60 \mathrm{~min}$ exposure time; therefore the possibility of higher elimination of protoscolices following longer exposure times remains as a subject which needs further investigations.

In the present study, glucose at $40 \%$ concentration had no significant effect on protoscolices at exposure times less than 20 minutes, but after 20,30, and $40 \mathrm{~min}$ this concentration of glucose killed $98.8 \%, 99.6 \%$, and $100 \%$ of protoscolices, respectively. So far, no study has been carried out regarding the effect of $40 \%$ glucose on protoscolices; therefore this concentration could be suggested as a suitable alternative protoscolicidal agent in future studies. Moreover, $50 \%$ glucose concentration in 1 to 5 min exposure times killed approximately $50 \%$ of protoscolices, and, in 6 to $8 \mathrm{~min}$, this effect was ascending, but, in 9 to $10 \mathrm{~min}$ exposure times, a significant amount of protoscolices died out. Hosseini et al. indicated that the significant effect on protoscolices occurred in 1 to 5 minutes exposure time [12]. In our study, after a $20 \mathrm{~min}$ exposure time, all protoscolices lost their viability, but in other studies it was mentioned that such effect happened after 15 and 30 minutes $[8,9]$. This difference in effectiveness could be related to diversity of parasite strains and differences in geographic location and experimental conditions; however, further studies are required.

Hypertonic glucose was initially used as a scolicidal agent on pericardial hydatid cyst of human by Ferrini et al. [10]. Moreover, Tejada et al. demonstrated that the use of hypertonic glucose produced no apparent symptoms [11]. In this regard, there are other studies in which $10 \%$ and $25 \%$ glucose solutions as well as normal saline were injected into the peritoneal cavity of rats and after 6, 24, and 48 hours, the peritoneal and mesenteric tissue of rats were examined for fibrosis and necrosis. Although glucose and normal saline solutions in the peritoneal cavity produced, to some extent, nonspecific inflammation but fibrosis or necrosis in the mesentery and parietal peritoneum was not seen and the degree of inflammation caused by $10 \%$ and $25 \%$ glucose and normal saline was equal [17]. In addition, Hosseini et al. in similar experiments following injection of $10 \%, 15 \%, 25 \%$, and $50 \%$ glucose concentrations into the gallbladder of rabbits showed that hypertonic glucose is a harmless substance and does not lead to the fibrosis of bile ducts and hepatic necrosis [18]. Therefore, it is suggested that hypertonic glucose could be a suitable replacement for lots of protoscolicidal materials with side effects.

On the other hand, hypertonic saline at $20 \%$ concentration, which destroys the wall of hydatid cyst by severe osmotic properties, is widely used today as a scolicidal agent [19]. In this study, hypertonic saline, compared with different concentrations of glucose, had a significant effect on the protoscolices within the first minutes of exposure time. Hypertonic saline, in our study, killed $97 \%$ of protoscolices in 10 minutes, but the total destruction of protoscolices was obtained after 20 minutes; however, this time course varies in different studies. In some other studies, the killing of all protoscolices occurred after 2, 4, 15, and $45 \mathrm{~min}$ exposure times $[8,13,14,20]$. Although $20 \%$ hypertonic saline is an effective protoscolicidal agent compared with the hypertonic glucose used in this study and other studies [13, 14, 20], nevertheless the dangerous side effects such as peritoneal inflammation, fibrosis of bile ducts, liver necrosis [14, 19], and the sharp rise in blood sodium have been reported after injection of hypertonic saline [21-23], and the use of alternative materials is required.

\section{Conclusion}

In the present study, although the effect of hypertonic saline on protoscolices was more than hypertonic glucose in lower exposure times, but the effect of both $20 \%$ hypertonic saline and $40 \%$ hypertonic glucose was $100 \%$ after 20 min exposure time. It seems that using hypertonic glucose, compared with hypertonic saline, can be preferred as a protoscolicidal agent. Therefore, our findings of in vitro study indicated that 40 and $50 \%$ hypertonic glucose could be an appropriate protoscolicidal agent for surgical operations; however to support in vitro findings, in vivo studies and further investigations are suggested. 


\section{Conflict of Interests}

The authors declare that there is no conflict of interests regarding the publication of this paper.

\section{Acknowledgments}

This study was supported by a fund from the Qazvin University of Medical Sciences, Qazvin, Iran.

\section{References}

[1] O. Topcu, Z. Sumer, E. Tuncer, C. Aydin, and A. Koyuncu, "Efficacy of chlorhexidine gluconate during surgery for hydatid cyst," World Journal of Surgery, vol. 33, no. 6, pp. 1274-1280, 2009.

[2] G. Adas, S. Arikan, O. Kemik, A. Oner, N. Sahip, and O. Karatepe, "Use of albendazole sulfoxide, albendazole sulfone, and combined solutions as scolicidal agents on hydatid cysts (in vitro study)," World Journal of Gastroenterology, vol. 15, no. 1, pp. 112-116, 2009.

[3] M. A. Rajabi, "Fatal reactions and methaemoglobinaemia after silver nitrate irrigation of hydatid cyst," Surgical Practice, vol. 13, no. 1, pp. 2-7, 2009.

[4] K. Puryan, K. Karadayi, O. Topcu et al., "Chlorhexidine gluconate: an ideal scolicidal agent in the treatment of intraperitoneal hydatidosis?" World Journal of Surgery, vol. 29, no. 2, pp. 227-230, 2005.

[5] M. Moazeni and M.-R. Alipour-Chaharmahali, "Echinococcus granulosus: in vitro effectiveness of warm water on protoscolices," Experimental Parasitology, vol. 127, no. 1, pp. 14-17, 2011.

[6] D. P. McManus, W. Zhang, J. Li, and P. B. Bartley, "Echinococcosis," The Lancet, vol. 362, no. 9392, pp. 1295-1304, 2003.

[7] E. Tozar, O. Topcu, K. Karayalcin, S. I. Akbay, and S. Hengirmen, "The effects of cetrimide-chlorhexidine combination on the hepato-pancreatico-biliary system," World Journal of Surgery, vol. 29, no. 6, pp. 754-758, 2005.

[8] R. Caglar, M. F. Yuzbasioglu, E. Bulbuloglu, M. Gul, F. Ezberci, and I. T. Kale, "In vitro effectiveness of different chemical agents on scolices of hydatid cyst," Journal of Investigative Surgery, vol. 21, no. 2, pp. 71-75, 2008.

[9] A. Beguiristain, B. Sesma, F. Ponce et al., "An evaluation of the in vitro scolicide activity of hydrogen peroxide, povidone-iodine, glucose solution, albendazole and albendazole sulfoxide against Echinococcus granulosus," Research in Surgery, vol. 6, no. 1, pp. $1-5,1994$.

[10] J.-B. Ferrini, L. Pichard, J. Domergue, and P. Maurel, "Longterm primary cultures of adult human hepatocytes," ChemicoBiological Interactions, vol. 107, no. 1-2, pp. 31-45, 1997.

[11] J. G. Tejada, J. Saavedra, L. Molina, A. Forteza, and C. Gomez, "Hydatid disease of the interventricular septum causing pericardial effusion," Annals of Thoracic Surgery, vol. 71, no. 6, pp. 2034-2035, 2001.

[12] S. V. Hosseini, K. Ghanbarzadeh, J. Barzin, S. M. Sadjjadi, N. Tanideh, and D. Mehrabani, "In vitro protoscolicidal effects of hypertonic glucose on protoscolices of hydatid cyst," The Korean Journal of Parasitology, vol. 44, no. 3, pp. 239-242, 2006.

[13] M. Moazeni, M. J. Saharkhiz, A. A. Hoseini, and A. M. Alavi, "In vitro scolicidal effect of Satureja khuzistanica (Jamzad) essential oil," Asian Pacific Journal of Tropical Biomedicine, vol. 2, no. 8, pp. 616-620, 2012.
[14] C. Kayaalp, M. Balkan, C. Aydin et al., "Hypertonic saline in hydatid disease," World Journal of Surgery, vol. 25, no. 8, pp. 975979, 2001.

[15] B. Kilicoglu, K. Kismet, S. S. Kilicoglu et al., "Effects of honey as a scolicidal agent on the hepatobiliary system," World Journal of Gastroenterology, vol. 14, no. 13, pp. 2085-2088, 2008.

[16] K. Kismet, S. S. Kilicoglu, B. Kilicoglu et al., "The effects of scolicidal agent propolis on liver and biliary tree," Journal of Gastrointestinal Surgery, vol. 12, no. 8, pp. 1406-1411, 2008.

[17] J. C. F. de Carvalho, A. T. Leal, L. F. de Sousa, and B. H. Filho, "Hypertonic glucose solution $10 \%-25 \%$ on the mesenterium and peritoneum of the rat: macroscopic and microscopic study," Acta Cirúrgica Brasileira, vol. 20, no. 6, pp. 455-460, 2005.

[18] S. V. Hosseini, G. R. Motazedian, D. Mehrabani et al., "Instillation of hypertonic glucose into gall bladder of rabbit: does it induce sclerosing cholangitis?" Journal of Applied Animal Research, vol. 29, no. 1, pp. 73-76, 2006.

[19] H. Besim, K. Karayalcin, O. Hamamci, C. Gungor, A. Korkmaz, and R. S. Stubbs, "Scolicidal agents in hydatid cyst surgery," $H P B$ Surgery, vol. 10, no. 6, pp. 347-351, 1998.

[20] K. H. Tappeh, A. Einshaei, R. Mahmudloo, H. Mohammadzadeh, M. Tahermaram, and S. J. Mousavi, "Effect of different concentrations of hypertonic saline at different times on protoscoleces of hydatid cyst isolated from liver and lung," Türkiye Parazitolojii Dergisi, vol. 35, no. 3, pp. 148-150, 2011.

[21] J. E. J. Krige, A. J. W. Millar, H. Rode, and D. Knobel, "Fatal hypernatraemia after hypertonic saline irrigation of hepatic hydatid cysts," Pediatric Surgery International, vol. 18, no. 1, pp. 64-65, 2002.

[22] J. M. Ramia Angel, E. Sancho Calatrava, J. M. Santos Blanco, F. Garrido Menendez, and R. Lozoya Albacar, "Hypernatraemia after hepatic hydatidosis surgery," Cirugia Espanola, vol. 86, no. 2, pp. 115-116, 2009.

[23] M. Mozeni and A. Nazer, "In vitro lethal effect of Zingiber officinale R. on protoscolices of hydatid cyst from sheep liver," Microbiology Research, vol. 2, no. 2, pp. 91-94, 2011. 

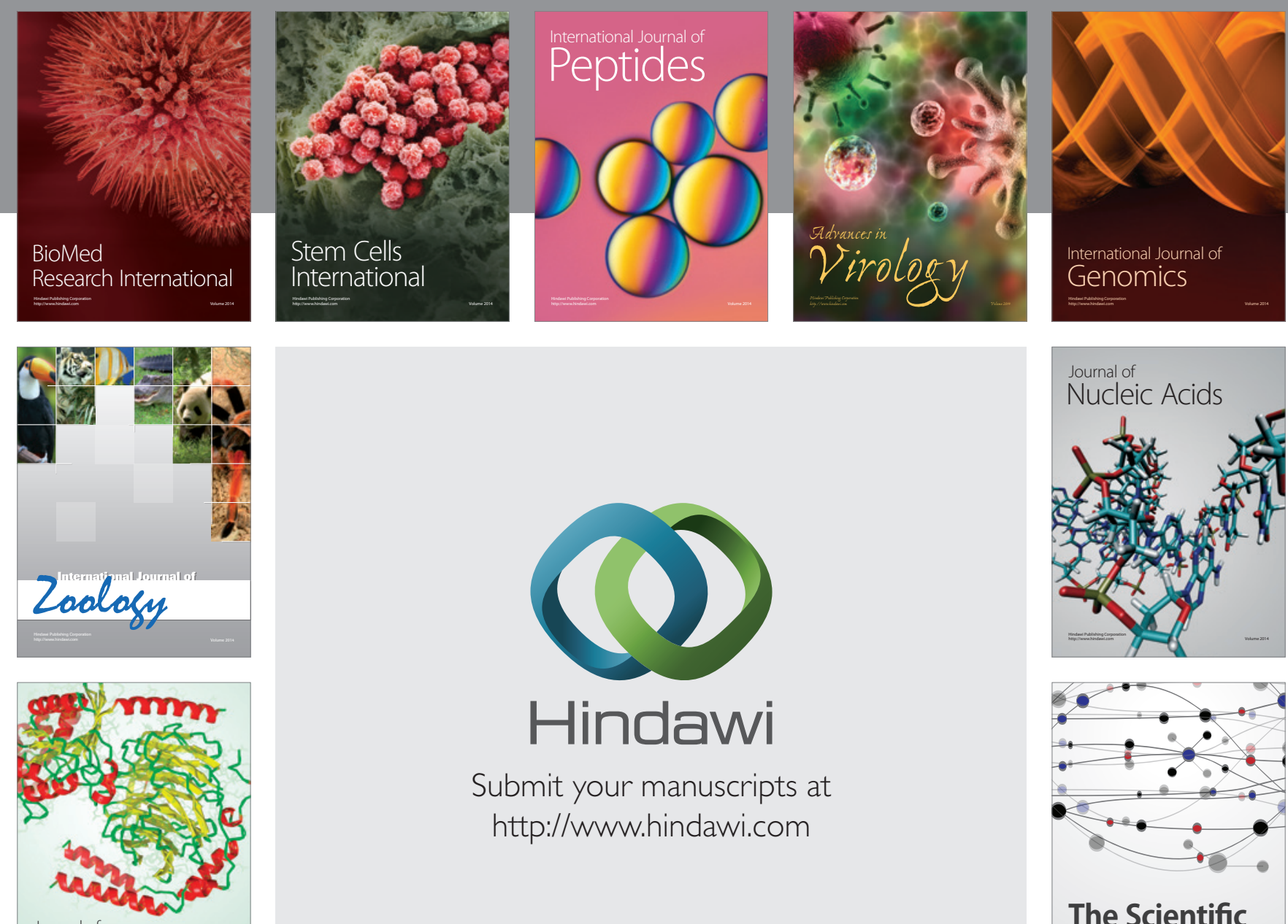

Submit your manuscripts at

http://www.hindawi.com

Journal of
Signal Transduction
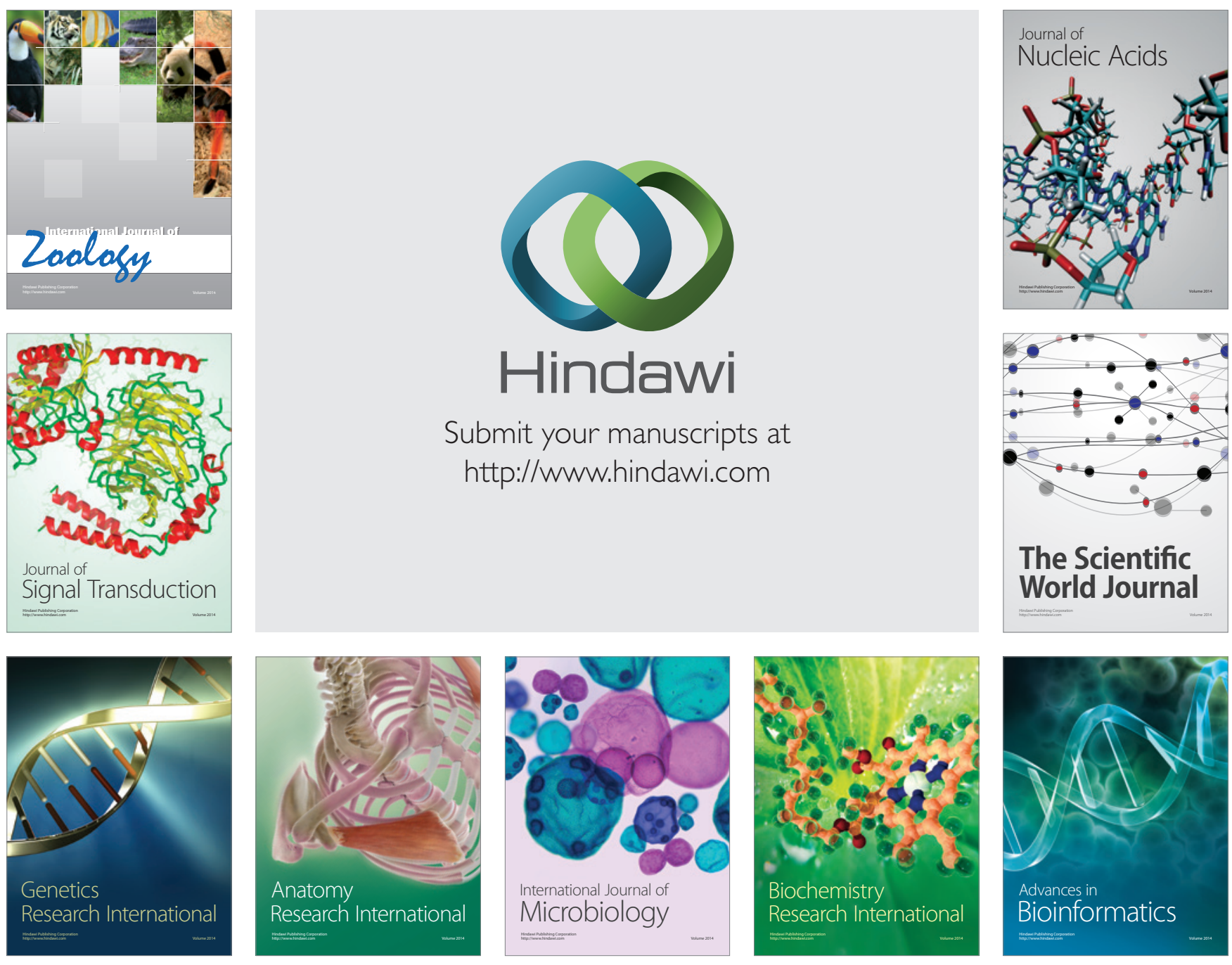

The Scientific World Journal
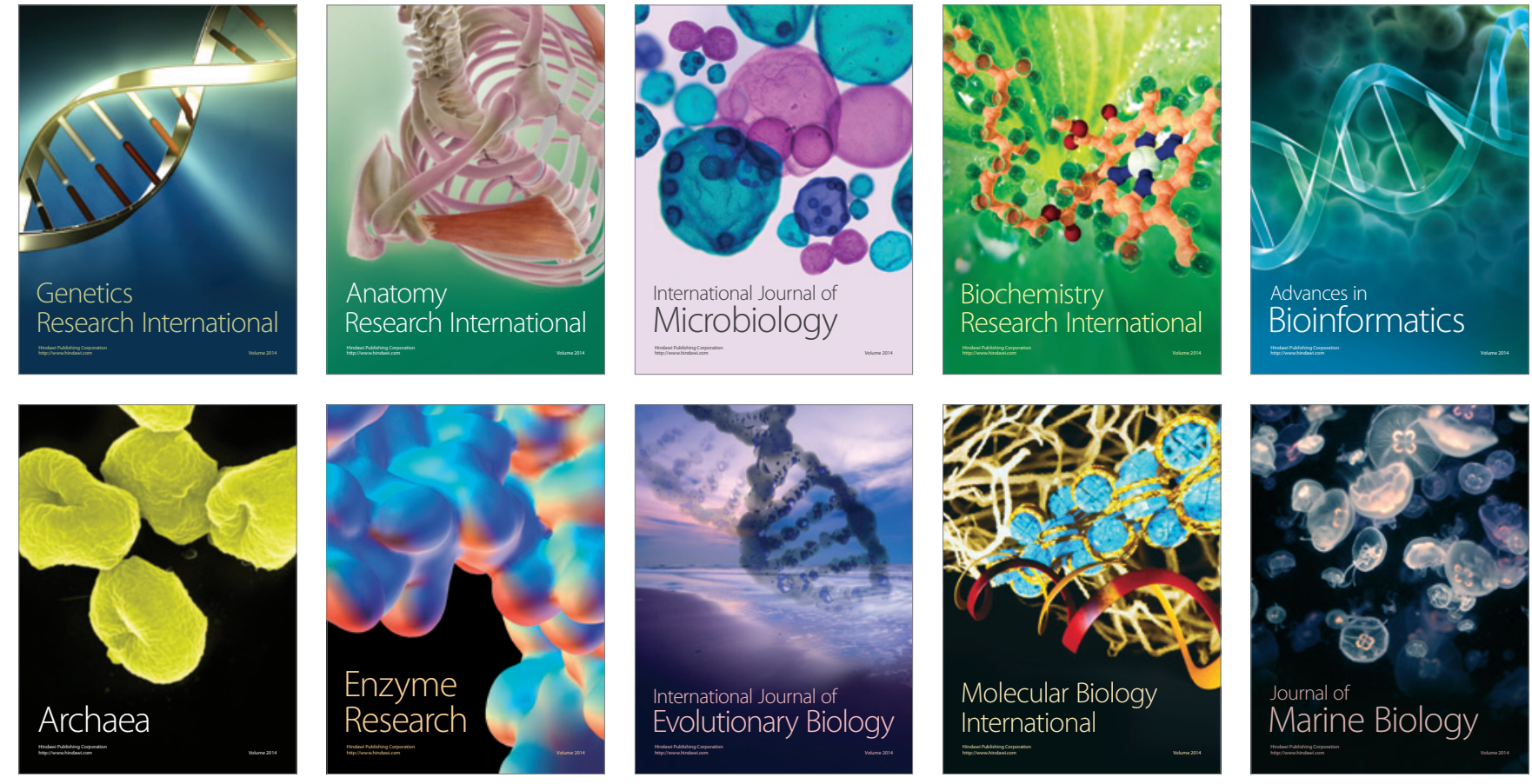